\title{
Basic reproductive number for a general hybrid epidemic model
}

\author{
Shujing Gao ${ }^{1 *}$, Yunbo Tu ${ }^{1}$ and Jialin Wang ${ }^{1}$
}

${ }^{*}$ Correspondence:
gaosjmath@126.com
${ }^{1}$ Key Laboratory of Jiangxi Province
for Numerical Simulation and
Emulation Techniques, Gannan
Normal University, Ganzhou, P.R.
China

"Correspondence: gaosjmath@126.com for Numerical Simulation and

\begin{abstract}
In this paper, a general hybrid epidemic model with multiple and non-periodic pulses in an environmental period is investigated. The definition and computation for the basic reproductive number $R_{0}$ are established. The published periodic research model (Yang, Xiao in Nonlinear Anal., Real World Appl. 52:224-234, 2012) can be considered as a special case of the new established model.
\end{abstract}

Keywords: Hybrid system; Basic reproductive number; Next infection operator

\section{Introduction}

The basic reproductive number $R_{0}$ is a measure of how many secondary hosts will become infected if one initial host is infected in a naïve population [2]. In the past decades, $R_{0}$ became one of the most important key parameters in mathematical epidemiology and was widely used in the study of epidemiology and within-host pathogen dynamics [3, 4]. It was established firstly in 1925 by Dublin and Lotka [5] and introduced as the "net reproduction rate" in human demography. For multistate stable population model, Inaba [6] defined $R_{0}$ as a spectral radius of the net reproductive matrix. Based on the next generation operator, Diekmann et al. [7] defined $R_{0}$ for heterogeneous population in a constant environment. For autonomous epidemic models, Van den Driessche and Watmough [8] investigated a method to calculate $R_{0}$ as the spectral radius of the next generation matrix. In 2006, Bacaër and Guernaoui [9] extended $R_{0}$ to the case of a periodic environment. Furthermore, Wang and Zhao [10] took the calculation of $R_{0}$ given in [8] for periodic systems as the spectral radius of the next infection operator. Based on the main results in [11], Wang and Zhao [12] defined the next infection operator as $R_{0}$ for a nonlocal and time-delayed reactiondiffusion model of dengue fever. $R_{0}$ and its computation formulae for a large class of timedelayed compartmental population models in a periodic environment were set up in [13].

It is well known that many natural phenomena and human activities have exhibited impulsive effects on the field of epidemiology. Recently, impulsive control problems in the application of epidemics, such as pulse vaccination [14-16] and pulse culling (roguing) [17], have received tremendous attention by many authors. Based on the spectral radius of the next infection operator, Yang and Xiao [18] gave the definition of $R_{0}$ for general impulsive epidemic models with periodic pulses on both infected and uninfected compartments. And then they [1] established $R_{0}$ and its calculation. However, the pulses are applied with the same time interval. An interesting question arises how $R_{0}$ can be defined

(c) The Author(s) 2018. This article is distributed under the terms of the Creative Commons Attribution 4.0 International License (http://creativecommons.org/licenses/by/4.0/), which permits unrestricted use, distribution, and reproduction in any medium, provided you give appropriate credit to the original author(s) and the source, provide a link to the Creative Commons license, and indicate if changes were made. 
and calculated for impulsive epidemic models with pulses at various time intervals in an environmental period. The present research intends to establish the theory of $R_{0}$ for such hybrid models.

The paper is organized as follows. In the next section, some basic notations and useful results for a general linear hybrid system are given. In Sect. 3, the definition and computation of $R_{0}$ for a general hybrid epidemic model are established. In Sect. 4, two examples to show the application are presented. A brief conclusion is given in the last section.

\section{Some useful results for a general linear impulsive system}

In order to obtain $R_{0}$ for a general nonautonomous impulsive system, we firstly give some useful results for a general linear impulsive system. The homogeneous linear impulsive differential system is considered as follows:

$$
\begin{cases}\dot{x}(t)=A(t) x(t), & t \neq t_{k}, \\ x\left(t_{k}^{+}\right)=P_{k} x\left(t_{k}\right), & t=t_{k}, k \in \mathbb{N}, \mathbb{N}=\{0,1, \ldots\} \\ x\left(t_{0}^{+}\right)=x_{0}, & t_{0} \geq 0\end{cases}
$$

which satisfies the following three conditions.

$\left(\mathrm{C}_{1}\right) A(\cdot) \in \mathrm{PC}\left(\mathbb{R}, \mathbb{R}^{n \times n}\right), A(t+\omega)=A(t)$, here $\omega>0$ is the environmental period, PC means piecewise continuous.

$\left(C_{2}\right) P_{k} \in \mathbb{R}^{n \times n}, \operatorname{det} P_{k} \neq 0, P_{0}=I$, where $I$ is an $n \times n$ identity matrix, $t_{k}<t_{k+1}$, and $\lim _{k \rightarrow+\infty} t_{k}=+\infty$.

$\left(C_{3}\right)$ There exists a positive integer $q$ such that $P_{k+q}=P_{k}, t_{k+q}=t_{k}+\omega$ for any $k \in \mathbb{N}$.

Set $\Psi_{A}(t, s)(t \geq s)$ be the evolution operator of the linear $\omega$-periodic system

$$
\dot{x}(t)=A(t) x(t), \quad x \in \mathbb{R}^{n} .
$$

That is, for each $s \in \mathbb{R}$, the following equalities hold:

$$
\dot{\Psi}_{A}(t, s)=A(t) \Psi_{A}(t, s), \quad \Psi_{A}(s, s)=I,
$$

where $I$ is an $n \times n$ identity matrix.

For any matrix $B \in \mathbb{R}^{n \times n}$, let $r(B)$ be the spectral radius of $B$. Throughout this paper, we denote

$$
\Phi_{A P_{k}}(\omega):=\prod_{i=1}^{q}\left(P_{q-i+1} \Psi_{A}\left(t_{q-i+1}, t_{q-i}\right)\right)
$$

Since $\Phi_{A P_{k}}(\omega)$ is a nonnegative matrix, then $r\left(\Phi_{A P_{k}}(\omega)\right)$ is the principal eigenvalue of $\Phi_{A P_{k}}(\omega)$, in the sense that it is simple and admits an eigenvector $v^{*} \gg 0$, i.e., $v^{*} \in \operatorname{Int} \mathbb{R}_{+}^{n}$. Some useful results for model (2.1) are below.

Theorem 2.1 If $\eta=(1 / \omega) \ln r\left(\Phi_{A P_{k}}(\omega)\right)$, then there exists a positive $\omega$-periodic function $v(t)$ such that $\exp (\eta t) v(t)$ is a solution of system (2.1). 
Proof Let $v^{*} \gg 0$ be an eigenvector associated with the principal eigenvalue $r\left(\Phi_{A P_{k}}(\omega)\right)$. By the change of variable $x(t)=\exp (\eta t) v(t)$, the impulsive linear system (2.1) is reduced to the following system:

$$
\begin{cases}\dot{v}(t)=A(t) v-\eta v=(A(t)-\eta I) v(t), & t \neq t_{k}, k \in \mathbb{Z}_{+} \\ v\left(t^{+}\right)=P_{k} v(t), & t=t_{k} . \\ v\left(t_{0}^{+}\right)=v^{*}, & t_{0} \geq 0 .\end{cases}
$$

For any $t>t_{0}$, there exist two nonnegative integers $l$ and $m(0 \leq m<q)$ such that $l \omega+t_{m}<$ $t \leq l \omega+t_{m+1}$. Solving (2.3), for $l \omega+t_{m}<t \leq l \omega+t_{m+1}$, we get

$$
\begin{aligned}
v(t)= & \Psi_{A-\eta I}\left(t, l \omega+t_{m}\right)\left(\prod_{i=1}^{m}\left(P_{m-i+1} \Psi_{A-\eta I}\left(t_{m-i+1}, t_{m-i}\right)\right)\right) \\
& \times\left(\Phi_{(A-\eta I) P_{k}}(\omega)\right)^{l} v^{*} .
\end{aligned}
$$

It is easy to see that

$$
\Phi_{(A-\eta I) P_{k}}(\omega)=\exp (-\eta \omega) \Phi_{A P_{k}}(\omega)
$$

From (2.4), (2.5), and $\eta=(1 / \omega) \ln r\left(\Phi_{A P_{k}}(\omega)\right)$, the following formula can be derived:

$$
\begin{aligned}
v\left((n+1) \omega^{+}\right) & =\left(\Phi_{(A-\eta I) P_{k}}(\omega)\right)^{n+1} v^{*} \\
& =\left(\Phi_{(A-\eta I) P_{k}}(\omega)\right)^{n} \exp (-\eta \omega) \Phi_{A P_{k}}(\omega) v^{*} \\
& =\left(\Phi_{(A-\eta I) P_{k}}(\omega)\right)^{n} \exp (-\eta \omega) r\left(\Phi_{A P_{k}}(\omega)\right) v^{*} \\
& =\left(\Phi_{(A-\eta I) P_{k}}(\omega)\right)^{n} v^{*} \\
& =v\left(n \omega^{+}\right) .
\end{aligned}
$$

Thus, $v(t)$ is a positive $\omega$-periodic solution of system (2.3) and $\exp (\eta t) v(t)$ is a solution of system (2.1). This completes the proof.

Theorem 2.2 If $r\left(\Phi_{A P_{k}}(\omega)\right)<1$, then the trivial solution of system (2.1) is asymptotically stable.

Using a method similar to that in [19], this result can be easily proved, not shown in this paper.

\section{The basic reproductive number for a general hybrid epidemic system}

Consider an impulsive differential system with non-periodic pulses in an environment period:

$$
\begin{cases}\dot{x}(t)=f(t, x), & t \neq t_{k}, t \in \mathbb{R} \\ \Delta x\left(t_{k}\right)=\Theta_{k}\left(x\left(t_{k}\right)\right), & t=t_{k}, k \in \mathbb{N} \\ x\left(t_{0}^{+}\right)=x_{0}, & t_{0} \geq 0,\end{cases}
$$


where $f: \mathbb{R}^{+} \times \Omega \rightarrow \mathbb{R}^{n}$ is an $\omega$-periodic vector function, here $\Omega \subseteq \mathbb{R}^{n}$ is an open set, $\Delta x\left(t_{k}\right)=x\left(t_{k}^{+}\right)-x\left(t_{k}\right), x\left(t_{k}^{+}\right)=\lim _{h \rightarrow 0^{+}} x\left(t_{k}+h\right)$.

The existence and uniqueness of the solution for system (3.1) can be found in [20].

We write $x=\left(x_{1}, x_{2}, \ldots, x_{n}\right)^{T}$, here $T$ denotes the transpose. For a compartmental epidemic model, we can split the compartments by two types with the first $m$ compartments $\left\{x_{1}, x_{2}, \ldots, x_{m}\right\}$ the infected individuals, and $\left\{x_{m+1}, x_{m+2}, \ldots, x_{n}\right\}$ the uninfected individuals. Denote $X=\left(x_{1}, \ldots, x_{m}\right), Y=\left(x_{m+1}, \ldots, x_{n}\right)$. Define

$$
X_{s}=\left\{x \geq 0 \mid x_{i}=0, i=1, \ldots, m\right\}
$$

Set $x\left(t_{k}^{+}\right)=x\left(t_{k}\right)+\Theta_{k}\left(x\left(t_{k}\right)\right):=\phi_{k}\left(x\left(t_{k}\right)\right)$, where $\phi_{k}: \Omega \rightarrow \Omega, \phi_{k} \in \mathbb{C}^{1}(\Omega, \Omega)$.

Taking the symbol in $[1,10]$, we can rewrite the vector field of $(3.1)$ as

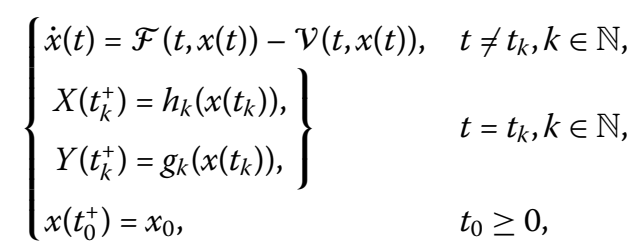

where $\mathcal{F}(t, x)$ is the newly infected rate, $\mathcal{V}$ represents the net transfer rate out of compartments, $\phi_{k}=\left(h_{k}, g_{k}\right)^{T}, \quad h_{k}=\left(\phi_{k 1}, \ldots, \phi_{k m}\right), \quad g_{k}=\left(\phi_{k(m+1)}, \ldots, \phi_{k n}\right)$. We assume that system (3.2) has a disease-free periodic solution $x^{*}(t)=\left(0, \ldots, 0, x_{m+1}^{*}(t), \ldots, x_{n}^{*}(t)\right)$ over the time interval $(n \omega,(n+1) \omega]$ with $x_{i}^{*}(t)>0, m+1 \leq i \leq n$, for all $t$. In addition, eight assumptions, similar to those by Yang and Xiao [1], are made as follows:

$\left(\mathrm{H}_{1}\right)$ If $x_{i} \geq 0$, then the functions $\mathscr{F}_{i}(t, x), \mathcal{V}_{i}^{+}(t, x)$, and $\mathcal{V}_{i}^{-}(t, x)$ are nonnegative and continuous on $R \times R_{+}^{n}$ and continuously differential with respect to $x$ for $i=1, \ldots, n$.

$\left(\mathrm{H}_{2}\right)$ If $x_{i}=0$, then $\mathcal{V}_{i}^{-}=0$. Particularly, if $x \in X_{s}$, then $\mathcal{V}_{i}^{-}=0$ for $i=1, \ldots, m$.

$\left(\mathrm{H}_{3}\right) \mathcal{F}_{i}=0$ if $i>m$.

$\left(\mathrm{H}_{4}\right)$ If $x \in X_{s}$, then $\mathcal{F}_{i}(x)=\mathcal{V}_{i}^{+}(x)=0$ for $i=1, \ldots, m$.

$\left(\mathrm{H}_{5}\right)$ The pulses on the infected compartments must be uncoupled with the uninfected compartments, that is, $h_{k}\left(x\left(t_{k}\right)\right)$ is essentially $h_{k}\left(X\left(t_{k}\right)\right)$.

$\left(\mathrm{H}_{6}\right)$ For any positive integer $k$, there exists a positive integer $q$ such that $t_{k+q}=t_{k}+\omega$, $h_{k+q}(x)=h_{k}(x), g_{k+q}(x)=g_{k}(x)$, and $h_{k}(0)=0$.

$\left(\mathrm{H}_{7}\right) r\left(\Phi_{M Q_{k}}(\omega)\right)<1$, where $\Phi_{M Q_{k}}(\omega):=\prod_{i=1}^{q}\left(Q_{q-i+1} \Phi_{M}\left(t_{q-i+1}, t_{q-i}\right)\right)$, and $\Phi_{M Q_{k}}(t)$ is the fundamental solution matrix of the following impulsive system:

$$
\begin{cases}\dot{Z}(t)=M(t) Z, & t \neq t_{k}, k \in \mathbb{N}, \\ Z\left(t_{k}^{+}\right)=Q_{k} Z\left(t_{k}\right), & t=t_{k}, k \in \mathbb{N},\end{cases}
$$

where

$$
M(t)=\left(\frac{\partial f_{i}\left(t, x^{*}(t)\right)}{\partial x_{j}}\right)_{m+1 \leq i, j \leq n}, \quad Q_{k}=\left(\frac{\partial \phi_{k i}\left(x^{*}(t)\right)}{\partial x_{j}}\right)_{m+1 \leq i, j \leq n} .
$$

$\left(\mathrm{H}_{8}\right) r\left(\Phi_{-V P_{k}}(\omega)\right)<1$, where

$$
V(t)=\left(\frac{\partial \mathcal{V}_{i}\left(t, x^{*}(t)\right)}{\partial x_{j}}\right)_{1 \leq i, j \leq m}, \quad P_{k}=\left(\frac{\partial \phi_{k i}\left(x^{*}(t)\right)}{\partial x_{j}}\right)_{1 \leq i, j \leq m} .
$$


We let

$$
F(t)=\left(\frac{\partial \mathcal{F}_{i}\left(t, x^{*}(t)\right)}{\partial x_{j}}\right)_{1 \leq i, j \leq m} .
$$

In the following, we study the threshold dynamics of model (3.1). It indicates that its basic reproductive number can be defined as the spectral radius of the next infection operator as that in periodic environments, see [10].

Apparently, the matrices $P_{k}$ and $Q_{k}(k \in \mathbb{N})$ are constant matrices. $F(t)$ is nonnegative, and $-V(t)$ is cooperative in the sense that the off-diagonal elements of $-V(t)$ are nonnegative.

Denote $Y(t, s)$ to be the evolution operator of the following system:

$$
\begin{cases}\dot{y}(t)=-V(t) y, & t \neq t_{k}, k \in \mathbb{N} \\ y\left(t_{k}^{+}\right)=P_{k} y\left(t_{k}\right), & t=t_{k}, k \in \mathbb{N} .\end{cases}
$$

From assumption $\left(\mathrm{H}_{8}\right)$ and Theorem 2.2, we know that the trivial solution of the impulsive differential system (3.5) is asymptotically stable.

Define $C_{\omega}$ as the ordered Banach space of all $\omega$-periodic functions from $\mathbb{R}$ to $\mathbb{R}^{m}$, which is equipped with maximum norm $\|\cdot\|_{\infty}$. Denote $C_{\omega}^{+}=\left\{\phi \in C_{\omega}: \phi(t) \geq 0, t \in \mathbb{R}\right\}$ as the initial distribution of infectious individuals. Following the idea in [1], we define the next infection operator $L$ :

$$
(L \phi)(t)=\lim _{a \rightarrow-\infty} \int_{a}^{t} Y(t, s) F(s) \phi(s) d s, \quad \forall t \in \mathbb{R}, \phi \in C_{\omega}^{+} .
$$

Obviously, the next infection operator $L$ is well defined, positive, continuous, and compact on the domain. Then we define the basic reproductive number of (3.2) as the spectral radius of $L$, that is,

$$
R_{0}=r(L)
$$

In order to calculate $R_{0}$, we consider an auxiliary linear impulsive system with nonperiodic pulses in an environmental period

$$
\begin{cases}\dot{U}(t)=\left[-V(t)+\frac{F(t)}{\lambda}\right] U(t), & t \neq t_{k}, k \in \mathbb{N}, \\ U\left(t^{+}\right)=P_{k} U(t), & t=t_{k}, k \in \mathbb{N},\end{cases}
$$

where $\lambda \in(0, \infty)$. Let $U(t, s, \lambda), t \geq s, s \in R$ be the evolution operator of system (3.7). Thus $U(\omega, 0, \lambda)=\Phi_{\left(\frac{F}{\lambda}-V\right) P_{k}}(\omega)$.

By the constant-variation formula for linear impulsive periodic equations (see [10]), we can prove the following result and obtain a numerical method to calculate the basic reproductive number for the hybrid epidemic system (3.1).

Theorem 3.1 Assume that $\left(\mathrm{H}_{1}\right)-\left(\mathrm{H}_{8}\right)$ hold, we have the following statements.

(i) If $r\left(\Phi_{\left(\frac{F}{\lambda}-V\right) P_{k}}(\omega)\right)=1$ has a positive solution $\lambda_{0}$, then $\lambda_{0}$ is an eigenvalue of $L$, and hence $R_{0}>0$. 
(ii) If $R_{0}>0$, then $\lambda=R_{0}$ is the unique solution of $r\left(\Phi_{\left(\frac{F}{\lambda}-V\right) P_{k}}(\omega)\right)=1$.

(iii) $R_{0}=0$ if and only if $r\left(\Phi_{\left(\frac{F}{\lambda}-V\right) P_{k}}(\omega)\right)<1$ for all $\lambda>0$.

According to the results of Theorem 3.1, we obtain that $R_{0}$ for the general hybrid epidemic model (3.2) is the positive solution of $r\left(\Phi_{\left(\frac{F}{\lambda}-V\right) P_{k}}(\omega)\right)=1$. In the following, we show that the basic reproductive number serves as a threshold value for the local stability of the disease-free periodic solution $x^{*}(t)$ of (3.2).

Theorem 3.2 Assume that $\left(\mathrm{H}_{1}\right)-\left(\mathrm{H}_{8}\right)$ hold. The following statements are valid:

(i) $R_{0}=1$ if and only if $r\left(\Phi_{(F-V) P_{k}}(\omega)\right)=1$.

(ii) $R_{0}>1$ if and only if $r\left(\Phi_{(F-V) P_{k}}(\omega)\right)>1$.

(iii) $R_{0}<1$ if and only if $r\left(\Phi_{(F-V) P_{k}}(\omega)\right)<1$.

The disease-free periodic solution $x^{*}(t)$ is asymptotically stable if $R_{0}<1$, and unstable if $R_{0}>1$.

Using a similar method of Theorem 2.2 in [10], this result can be easily proved, not shown in this paper.

Remark 3.1 In [1], Yang and Xiao considered a nonlinear impulsive system, in which the pulse period is equal to the environmental period. If we take $q=1$, then model (3.1) yields the model that was studied in [1]. From (2.2), we have $\Phi_{-V P_{k}}(\omega)=P_{1} \Psi_{-V}(\omega, 0)$ if $q=1$, which is consistent with the result of the literature. It indicates that here a more general model is established including the previous model as a special case.

\section{Examples}

We give two examples to illustrate the calculation of $R_{0}$ for impulsive nonautonomous model using Theorem 3.1.

Example 1 Barbour's schistosomiasis model with seasonal fluctuations was proposed and studied in [21]. Incorporating impulsive snail-killing strategy, we consider Barbour's schistosomiasis model with periodic infection rates and impulsive control strategy:

$$
\left\{\begin{array}{l}
\dot{M}(t)=a(t) \Delta y(1-M)-g M, \\
\dot{y}(t)=b(t) \frac{\Sigma}{\Delta} M(1-y)-\mu y, \\
M\left(t^{+}\right)=M(t), \\
y\left(t^{+}\right)=\left(1-\theta_{k}\right) y(t),
\end{array}\right\} t \neq t_{k}
$$

where $M$ and $y$ denote the prevalence of infection in humans and the prevalence of infection in snails, respectively. $a$ and $b$ are infection rates, $g$ is the recovery rate for definitive host infections, $\Delta$ and $\Sigma$ are the densities of infected snails and definitive hosts, respectively. $\mu$ is per capita death rate of infected snails. For snail control, we assume spraying pesticide twice a year, that is, $q=2$ and $\omega=1$ (year). We also assume that $t_{k+2}=t_{k}, \theta_{k+2}=\theta_{k}$, and $a(t)$ and $b(t)$ are nonnegative piecewise continuous functions with period 1 .

System (4.1) has a disease-free periodic solution $(0,0)$. For system (4.1), we have

$$
\mathcal{F}(t, x)=\left(\begin{array}{l}
a(t) \Delta y(1-M) \\
b(t) \frac{\Sigma}{\Delta} M(1-y)
\end{array}\right), \quad \mathcal{V}(t, x)=\left(\begin{array}{l}
g M \\
\mu y
\end{array}\right)
$$


Then, from (3.3) and (3.4), we get

$$
\begin{aligned}
& F(t)=\left(\begin{array}{cc}
0 & a(t) \Delta \\
b(t) \frac{\Sigma}{\Delta} & 0
\end{array}\right), \quad V(t)=\left(\begin{array}{ll}
g & 0 \\
0 & \mu
\end{array}\right), \\
& P_{1}=\left(\begin{array}{cc}
1 & 0 \\
0 & 1-\theta_{1}
\end{array}\right), \quad P_{2}=\left(\begin{array}{cc}
1 & 0 \\
0 & 1-\theta_{2}
\end{array}\right) .
\end{aligned}
$$

Obviously, assumptions $\left(\mathrm{H}_{1}\right)-\left(\mathrm{H}_{8}\right)$ hold. According to Theorem 3.1, we know that the basic reproductive number $R_{0}$ for system (4.1) is a solution of the polynomial $r\left(\Phi_{\left(\frac{F}{\lambda}-V\right) P_{k}}(\omega)\right)=1$, where

$$
\Phi_{\left(\frac{F}{\lambda}-V\right) P_{k}}(\omega)=P_{2} \Phi_{\frac{F}{\lambda}-V}\left(t_{2}, t_{1}\right) P_{1} \Phi_{\frac{F}{\lambda}-V}\left(t_{1}, t_{0}\right),
$$

and $\Phi_{\left(\frac{F}{\lambda}-V\right) P_{k}}(t, s)(t \geq s)$ is the evolution operator of the system

$$
\begin{cases}\dot{U}(t)=\left[\begin{array}{cc}
-g & \frac{a(t) \Delta}{\lambda} \\
b(t) \frac{\Sigma}{\Delta \lambda} & -\mu
\end{array}\right] U(t), & t \neq t_{k}, k \in \mathbb{N}, \\
U\left(t^{+}\right)=P_{k} U(t), & t=t_{k}, k \in \mathbb{N} .\end{cases}
$$

We use the same parameter values in [21] with system (4.1), which are $g=0.25, \mu=2$, $\Delta=133.5, \Sigma=0.017, a(t)=\frac{\pi}{2} a|\sin (\pi t)|, b(t)=\frac{\pi}{2} b|\sin (\pi t)|, a=0.621, b=49.056$. The control parameter values are chosen as $\theta_{1}=0.6, \theta_{2}=0.5, t_{0}=0.3, t_{1}=0.5$, by numerical calculation, we get $R_{0}=0.752$.

Example 2 We propose an SIRS epidemic model with pulse vaccination and seasonality, in which non-periodic pulses in an environmental period are considered.

$$
\left\{\begin{array}{l}
\dot{S}(t)=(\mu-(1-\sigma) \mu I)(1-p)-\frac{\beta(t) S I}{1+\alpha(t) S}-\mu S+\delta R, \\
\dot{I}(t)=\frac{\beta(t) S I}{1+\alpha(t) S}-\mu I-\gamma I+(1-\sigma) \mu I, \\
\dot{R}(t)=(\mu-(1-\sigma) \mu I) p+\gamma I-(\mu+\delta) R, \\
S\left(t^{+}\right)=\left(1-\theta_{k}\right) S(t), \\
I\left(t^{+}\right)=I(t), \\
R\left(t^{+}\right)=R(t)+\theta_{k} S(t),
\end{array}\right\} \quad t \neq t_{k}, k \in \mathbb{N},
$$

where $S, I$, and $R$ denote the number of susceptible, infected, and removed individuals, respectively. $\mu$ is the natural birth rate and death rate, $\sigma$ is a probability that a child is born from an infectious mother and is also susceptible, $\gamma$ and $\delta$ are the transmission rates, $p$ is the continuous first vaccination rate, $\frac{\beta(t) S I}{1+\alpha(t) S}$ is the saturation incidence, where $\beta(t)$ (contact rate) and $\alpha(t)$ are two periodic continuous functions with period $\omega, \theta_{k}$ is the vaccination rate at time $t=t_{k}$. We assume that there exists a positive integer $q$ such that $\theta_{k+q}=\theta_{k}$, $t_{k+q}=t_{k}+\omega$ for any $k \in \mathbb{N}$. Model (4.2) was proposed in [22] where the pulse vaccination is periodic. 
First, we can easily know that system (4.2) has a disease-free periodic solution $\left(S^{*}(t), 0\right.$, $R^{*}(t)$ ). And from the definition of $F(t), V(t)$, and the impulsive matrix $P_{k}$, we can get

$$
F(t)=\frac{\beta(t) S^{*}(t)}{1+\alpha(t) S^{*}(t)}, \quad V(t)=\gamma+\sigma \mu, \quad P_{k}=1 .
$$

It follows from Theorem 3.1 that the basic reproductive number $R_{0}$ is the solution of the polynomial $r\left(\Phi_{\left(\frac{F}{\lambda}-V\right) P_{k}}(\omega)\right)=1$, where $\Phi_{\left(\frac{F}{\lambda}-V\right) P_{k}}(\omega)=U(\omega, 0, \lambda)$, and $U(t, 0, \lambda)$ is the evolution operator of the system

$$
\dot{U}(t)=\left(-(\gamma+\sigma \mu)+\frac{\beta(t) S^{*}(t)}{\lambda\left(1+\alpha(t) S^{*}(t)\right)}\right) U(t) .
$$

Thus,

$$
r\left(\Phi_{\left(\frac{F}{\lambda}-V\right) P_{k}}(\omega)\right)=\exp \left(\int_{0}^{\omega}\left(\frac{\beta(t) S^{*}(t)}{\lambda\left(1+\alpha(t) S^{*}(t)\right)}-(\gamma+\sigma \mu)\right) d t\right) .
$$

Solving the following equation about $\lambda$ :

$$
\exp \left(\int_{0}^{\omega}\left(\frac{\beta(t) S^{*}(t)}{\lambda\left(1+\alpha(t) S^{*}(t)\right)}-(\gamma+\sigma \mu)\right) d t\right)=1,
$$

we have the basic reproductive number

$$
R_{0}=\frac{1}{\omega(\gamma+\sigma \mu)} \int_{0}^{\omega} \frac{\beta(t) S^{*}(t)}{1+\alpha(t) S^{*}(t)} d t
$$

This is consistent with the result of [22] when pulse intervals are equal.

\section{Conclusion}

In this paper, we have given the definition and calculation of the basic reproductive number $R_{0}$ for the hybrid epidemic model, in which multiple and non-periodic pulses in an environmental period are taken into consideration. It extends the results of the published research model [1]. Under assumptions $\left(\mathrm{H}_{1}\right)-\left(\mathrm{H}_{8}\right)$, we have established the criteria (i.e., $\left.R_{0}<1\right)$ to ensure the local stability of the disease-free periodic solution.

\section{Acknowledgements}

We would like to give special thanks to the anonymous referees and to the editor which gave us very useful suggestions, which helped us greatly to improve this work.

\section{Funding}

The research has been supported by the Natural Science Foundation of China (11561004) and the Natural Science Foundation of Jiangxi Province (20171BAB201006).

\section{Competing interests}

The authors declare that they have no competing interests.

\section{Authors' contributions}

The main idea and theoretical proof of this paper were proposed by SG and JW. Programming of numerical simulation was completed by YT. All authors read and approved the final manuscript.

\section{Publisher's Note}

Springer Nature remains neutral with regard to jurisdictional claims in published maps and institutional affiliations. 


\section{References}

1. Yang, Y., Xiao, Y.: Threshold dynamics for compartmental epidemic models with impulses. Nonlinear Anal., Real World Appl. 13(1), 224-234 (2012). https://doi.org/10.1016/j.nonrwa.2011.07.028

2. Taylor, R.A., Mordecai, E.A., Gilligan, C.A., Rohr, J.R., Johnson, L.R.: Mathematical models are a powerful method to understand and control the spread of Huanglongbing. PerrJ. - J. Life Environ. Sci. 4, Article ID e2642 (2016). https://doi.org/10.7717/peerj.2642

3. Zhang, T., Meng, X., Zhang, T.: Global dynamics of a virus dynamical model with cell-to-cell transmission and cure rate. Comput. Math. Methods Med. 2015, 45-48 (2015)

4. Fan, X., Wang, L., Teng, Z.: Global dynamics for a class of discrete SEIRS epidemic models with general nonlinear incidence. Adv. Differ. Equ. 2016, 123 (2016). https://doi.org/10.1186/s13662-016-0846-y

5. Dublin, L.I., Lotka, A.J.: On the true rate of natural increase: As exemplified by the population of the United States, 1920. J. Am. Stat. Assoc. 20(151), 305-339 (1925). https://doi.org/10.1080/01621459.1925.10503498

6. Inaba, H.: A semigroup approach to the strong ergodic theorem of the multistate stabile population process. Math. Popul. Stud. 1, 49-77 (1988)

7. Diekmann, O., Heesterbeek, J.A.P., Metz, J.A.J.: On the definition and the computation of the basic reproduction ratio $R_{0}$ in the models for infectious disease in heterogeneous populations. J. Math. Biol. 28, 365-382 (1990)

8. Van den Driessche, P., Watmough, J.: Reproductive numbers and subthreshold endemic equilibria for compartmenta models of disease transmission. Math. Biosci. 180, 29-48 (2002)

9. Bacaër, N., Guernaoui, S.: The epidemic threshold of vector-borne diseases with seasonality - the case of cutaneous leishmaniasis in Chichaoua, Morocco. J. Math. Biol. 53, 421-436 (2006)

10. Wang, W., Zhao, X.Q.: Threshold dynamics for compartmental epidemic models in periodic environments. J. Dyn. Differ. Equ. 20, 699-717 (2008)

11. Thieme, H.R.: Spectral bound and reproduction number for infinite-dimensional population structure and time heterogeneity. SIAM J. Appl. Math. 70, 188-211 (2009)

12. Wang, W., Zhao, X.Q.: A nonlocal and time-delayed reaction-diffusion model of dengue transmission. SIAM J. Appl. Math. 71, 147-168 (2011)

13. Zhao, X.Q.: Basic reproduction ratios for periodic compartmental models with time delay. J. Dyn. Differ. Equ. 29 67-82 (2017)

14. D'Onofrio, A.: On pulse vaccination strategy in the SIR epidemic model with vertical transmission. Appl. Math. Lett. $18,729-732(2005)$

15. D'Onofrio, A.: Stability properties of pulse vaccination strategy in SEIR epidemic model. Math. Biosci. 179, 57-72 (2002)

16. Shulgin, B., Stone, L., Agur, Z.: Pulse vaccination strategy in the SIR epidemic model. Bull. Math. Biol. 60, 1123-1148 (1998)

17. Tang, S., Xiao, Y., Robert, C.: Dynamical analysis of plant disease models with cultural control strategies and economic thresholds. Math. Comput. Simul. 80, 894-921 (2010)

18. Yang, Y., Xiao, Y.: The effects of population dispersal and pulse vaccination on disease control. Math. Comput. Model. 52, 1591-1604 (2010). https://doi.org/10.1016/j.mcm.2010.06.024

19. Samoilenko, A.M., Perestyuk, N.A.: Differential Equations with Impulsive Effect. Visca Skola, Kiev (1987)

20. Bainov, D., Simeonov, P.S.: Impulsive Differential Equations: Periodic Solutions and Applications. Monographs and Surveys in Pure and Applied Mathematics, vol. 66. Longman, Harlow; Wiley, New York (1993)

21. Gao, S., He, Y., Liu, Y., Yang, G., Zhou, X.: Field transmission intensity of Schistosoma japonicum measured by basic reproduction ratio from modified Barbour's model. Parasit Vectors 6, Article ID 141 (2013). https://doi.org/10.1186/1756-3305-6-141

22. Gao, S., Liu, Y., Nieto, J.J., Andrade, H.: Seasonality and mixed vaccination strategy in an epidemic model with vertical transmission. Math. Comput. Simul. 81, 1855-1868 (2011)

\section{Submit your manuscript to a SpringerOpen ${ }^{\circ}$ journal and benefit from:}

- Convenient online submission

- Rigorous peer review

- Open access: articles freely available online

- High visibility within the field

- Retaining the copyright to your article

Submit your next manuscript at $>$ springeropen.com 